\title{
Resveratrol and Puerarin loaded polymeric nanoparticles to enhance the chemotherapeutic efficacy in spinal cord injury
}

\section{Qimin Song ( $\sim$ StephanieRenatagHIOuO@yahoo.com )}

Linyi People's Hospital

\section{Wei Chen}

Linyi People's Hospital

\section{Zongqin Zhao}

Qingdao University

Shuchao Zhao

Qingdao Haici Hospital

Lizhong Zhang

Linyi People's Hospital

\section{Original article}

Keywords: Resveratrol, Puerarin, nanocarriers, nanoparticles, spinal cord ischemia, spinal cord injury

Posted Date: June 19th, 2020

DOl: https://doi.org/10.21203/rs.3.rs-29754/v1

License: (9) This work is licensed under a Creative Commons Attribution 4.0 International License. Read Full License 


\section{Abstract}

\section{Introduction:}

Spinal cord injury includes inflammation and apoptosis of neurons, which is difficult to cure by systemic drug administration. Administration of natural active compounds (resveratrol and also Puerarin) by advance drug delivery technology improves the patient's conditions.

Material and Methods: Oil-in-water emulsion method was utilized to prepare resveratrol as well as puerarin loaded PLGA nanoparticles. The nanoparticles were subjected to mean zeta potential, mean particle size, encapsulation efficiency as well as in vitro drug release studies. The biochemical parameters i.e. malondialdehyde (MDA), reduced glutathione (GSH), superoxide dismutase (SOD), advanced oxidation products (AOPP), catalase (CAT) and nitrite/nitrate levels were tested for the loaded nanoparticles. Reperfusion injury induced rats treated with $10 \mathrm{mg} / \mathrm{kg}$ resveratrol and puerarin loaded nanoparticles protects spine from ischemia injury and supports biological parameters.

Results: The mean particle size varies from $238 \mathrm{~nm}$ to $274 \mathrm{~nm}$ and also particle size distribution was mono-dispersed ( 0.239 to 0.318 ). Zeta potential value of nanoparticles was observed to be $-12.6 \pm$ $2.1 \mathrm{mV}$. Optimized nanoparticles reveals $72 \%-79 \%$ of drug release over $36 \mathrm{~h}$ by diffusion mechanism. Significantly, lowers the levels of plasma nitrite/nitrate level as well as phosphorylation of p38MAPK pathways in reperfusion injury induced rats.

Conclusion: The resveratrol and puerarin loaded nanoparticles decreases free radicals produced by reperfusion injury induced rats, as well as decrease of oxidative stress because of IRI. Resveratrol and puerarin loaded nanoparticles decreases GSH, SOD and CAT antioxidant level, which helps in overall health improvement of patients.

\section{Introduction}

Spinal cord connected with brain by peripheral tissue and nervous system[1]. Spinal cord primarily comprise of neurons, glial cells, astrocytes and oligodendrocytes cells. Spinal neurons, poses cutaneous sensory input are mainly located in the dorsal spinal cord and interconnected in circuits; while neurons responsible for motor control are located in ventral spinal cord. The loss of neurons and axonal degeneration occurs at the lesion site due to spinal cord injury. In some cases severe functional impairment, paraplegia, or tetraplegia may occurs due to motor deficits, loss of sensory inputs and chronic pain [1,2]. Secondary spinal cord injury is a degenerative response that includes inflammation, ionic imbalance, excitotoxicity, neurotransmitter accumulation and apoptosis [3, 4]. The severity of the secondary injury response slowly to systemically administered drug within therapeutic window.

Pain relief, spasticity treatment, rehabilitation and prevention of complications are most focus area in long term therapy for spinal cord injury. The glial scars are developed in the trauma region, which limits the regenerative capacity of central nervous system. [5-7]. Special methods are required to reconstruct 
grey and white matter of CNS, which helps in promote axonal growth and reduce scar formation. [8]. The nanoparticles facilitate passage through the blood-spinal cord barrier and extend therapeutic delivery and/or imaging in the brain and spinal cord to protect it from toxins.

Resveratrol (3,5,4'-trihydroxy-trans-stilbene) is a polyphenol has antioxidant and anti-inflammatory activity $[9,10]$. Resveratrol helps in free radical scavenging, increases the antioxidant enzymes, promote neuroprotection and neutralizes oxidative stress. Puerarin (Daidzein-8-C-glucoside) is the major bioactive ingredient in kudzuroot [8]. Many researchers show that Puerarin has anti-apoptotic, anti-oxidant [11], anti-inflammatory [2], anti-arrhythmic[1], and anti-fibrosis [12] activity. These therapeutic effects of Resveratrol and Puerarin have much useful for treatment of spinal cord injury [13]. The major constrains for oral administration of Resveratrol and Puerarin orally is its short half-life, in which it is rapidly metabolized by first pass metabolism in liver. The nanocarriers useful for significant prolong residence time of the dosage; improve the efficacy of drugs as potential treatment of spinal cord. The main objective of this work was to evaluate the capability of Resveratrol and Puerarin encapsulated in Poly(DLlactic-co-glycolic acid) nanoparticles as treatment to reduce the oxidative stress in spinal cord injury [14].

\section{Materials And Methods 2.1. Materials}

Resveratrol (RES) and Puerarin (PUE) purchased from Sigma Aldrich (St. Louis, MO, 454 USA). Poly(lacticco-glycolic acid) with 50:50 ratio was purchased from Sigma-Aldrich. Polyvinyl alcohol, $6 \mathrm{~kg} / \mathrm{mol}$ and $80 \% \mathrm{~mol}$ hydrolyzed, was purchased from Polysciences Inc. (Warrington,USA). Acetonitrile (HPLC grade) was obtained from BDH Laboratory Supplies (Pools, UK). All chemicals were of analytical grade and were used as received. DMSO, ethylene diamine tetraacetic acid (EDTA) were purchased from Merck co. (Darmstadt, Germany). All other chemical regents were of analytical grade. All buffers were made with ultrapure water collected from Millipore at $18 \mathrm{M} \Omega$ resistances (Millipore, Bedford, USA).

\subsection{Animals}

Male wistar albino rats weighing 210-250 g were purchased and acclimatised to laboratory environment by exposed them to free access of food and water for $24 \mathrm{hrs}$. Prior to run the animal experimentation, all the procedures were taken to account for consideration and supervision by the Animal ethical clearance committee.

\subsection{Preparation of RES-PUE-PLGA nanoparticles}

Oil-in-water emulsion techniques were used for the drug loading in PLGA nanoparticles and PVA used as co-solvent to stabilized the emulsion. RES-PUE loaded nanoparticles were prepared with aqueous outer phase (PVA and $\mathrm{NaCl})$ covers the inner organic phase of PLGA $(0.25 \mathrm{dL} / \mathrm{g})$, Resveratrol (RES) and Puerarin (PUE) in DCM. Micrio-tip probe sonicator at 39 W (VCX750, Sonics and materials Inc, USA) was use as energy source for emulsion preparation over ice bath for $20 \mathrm{~s}$. The nanoparticles formed in emulsion were stabilized by addition of $40 \mathrm{~mL}$ of PVA solution $(25 \mathrm{mg} / \mathrm{mL})$ and stirred for $18 \mathrm{hrs}$ at room 
temperature. The RES-PUE nanoparticles were collected by ultracentrifugation at 40,000 rpm for 20 min at $4{ }^{\circ} \mathrm{C}$ (Sorvall-WX, ThermoFisher Scientific, USA), washed thrice with ultrapure water to remove free drugs and unbound PVA, lyophilized for 2 days $\left(-80^{\circ} \mathrm{C}\right.$ and $<10 \mu \mathrm{m}$ mercury pressure, Lab-conco Lyophilizer, USA) to get the powdered NPs.

To prepare an optimum dosage form, with surplus entrapment, the impact of drug to polymer ratio was studied. Herein, all the variables were found to be constant except that the drug to PLGA ratio showed variations by $20,30,40,50$ and $60 \%$ (w/w) on dry weight basis.

\subsection{Characterization of Resveratrol and Puerarin loaded nanoparticles (Res-Pue nanoparticles)}

RES-PUE loaded nanoparticles synthesis was optimized for Drug-polymer ratio, and thus encapsulation and thus encapsulation efficiency varied. Yield of nanoparticle was calculated from the weight of PLGA nanoparticles recovered, divided by the initial weight of PLGA taken for formulation $[15,16]$.

\subsection{Drug encapsulation efficiency (DE)}

Encapsulation efficiency was calculated from the ratio of theoretical drug content to fraction of drug detected per unit mass of particle. RES-PUE loaded nanoparticles sample (100 mg) was dissolved in $1 \mathrm{~mL}$ of HPLC grade methanol in a microcentrifuge tubes. The solution was mix for 5 min by vortexing (MX-S, Vortex mixer, Biobase) followed by centrifugation (BKC-TH20RL, Biobase) at 35,000 rpm for $20 \mathrm{~min}$ at $4{ }^{\circ} \mathrm{C}$ to collect the supernatant. The collected supernatant was filtered through $0.2 \mu \mathrm{m}$ syringe filter and $20 \mu \mathrm{l}$ was injected to RP-HPLC (e2695 separation module, Water's Alliance, Milford, MA, USA). The area under the curve (AUC) and retention time (tR) of the peaks were recorded by extracting the chromatogram at $270 \mathrm{~nm}$ and $304 \mathrm{~nm}$ wavelength for Resveratrol and Puerarin respectively, using operating software Empower3 pro. Resveratrol and Puerarin encapsulation efficiency and loading capacity was calculated using regression equation obtained from concentration vs AUC of standard Resveratrol and Puerarin respectively.

\subsection{Size of Particles and Zeta Potential of RES-PUE loaded nanoparticles}

The particle size analyzer (Zetasizer, Malvern Panalytical, UK) based on dynamic light scattering technique used for average particle size determination. Zeta potential of nanoparticles shows surface properties of nanoparticles and it was measured by electrostatic or charge repulsion/attraction between particles (Nano ZS analyzer, Malvern Panalytical, UK). Briefly RES-PUE loaded nanoparticles sample solution was prepared with water and solution was placed in separate polystyrene cuvettes meant for particle size and zeta potential measurement.

\subsection{Scanning Electron Microscopy}

Surface morphological evaluation of RES-PUE loaded nanoparticles werecarried out by scanning electron microscope (EVO, ZEISS Germany) for which lyophilized powder samples were spread on the carbon 
adhesive sample holder and coated with gold and used in scanning electron microscope[14].

\subsection{Atomic Force Microscopy}

Atomic force microscope (AFM) (Axio Observer Z1, Carl Zeiss Microscopy GmbH) was used for visualization of individual as well as groups of particles. It offers visualization in three dimensions, unlike other microscopy techniques. The samples were dispersed in optimum quantity of deionized water by sonication. Small amount of the dilution were put on a cleaned mica plate and incubated to dryness by a stream of nitrogen. This method was followed for all samples.

\subsection{Fourier Transmission Infrared spectroscopy (FTIR) analysis}

The chemical integrity of the polymer and drug was analysed via Fourier Transmission Infrared Spectroscopic technique (SPECTRUM RX I, Perkin Elmer, USA). Approximately $2 \mathrm{mg}$ of native Resveratrol, Puerarin and RES-PUE loaded nanoparticles samples were mixed separately in 300-400 mg of anhydrous $\mathrm{KBr}$ and ground properly in a mortar pestle. The sample mixture was compressed by applying hydraulic pressure of $2000 \mathrm{Kg} / \mathrm{cm}^{2}$ (Jasco MP2 mini press) for $2 \mathrm{~min}$. The FTIR spectrum was obtained by scanning all samples under $2 \mathrm{~cm}^{-1}$ resolution power in $4000-400 \mathrm{~cm}^{-1}$ spectral [17].

\subsection{Differential Scanning Calorimetry}

The physico-chemical properties and drug polymer compatibility of the drugs in the polymer were analysed via Differential Scanning Calorimetric technique (DSC-60, Shimadzu, Japan). The samples RESPUE loaded nanoparticles, native Resveratrol native Puerarin (2-4 mg) were sealed separately in standard aluminium pans and scanned $10^{\circ} \mathrm{C} / \mathrm{min}$ rate of heat supply at a temperature ranging from $50-$ $350^{\circ} \mathrm{C}$ with continuous nitrogen gas flow of $65 \mathrm{~mL} / \mathrm{min}$ [17].

\subsection{In-vitro drug release study}

RES-PUE loaded nanoparticles, native Resveratrol and Puerarin (each containing $5 \mathrm{mg}$ of Resveratrol and Puerarin) were kept in two separate hermetically sealed dialysis bags (12000-14000 Dalton, SigmaAldrich). The dialysis bags were hanged in a beaker that was filled with saline buffer of phosphate, maintained at $\mathrm{pH}=7.4(100 \mathrm{~mL})$ as dissolution medium maintained at $37 \pm 2{ }^{\circ} \mathrm{C}$ with continues stirring at $100 \mathrm{rpm}$. The entire assembly was covered with aluminium foil to prevent loss of dissolution media. $1 \mathrm{~mL}$ of samples was collected at specified time intervals. The release media was replenished with $1 \mathrm{~mL}$ of fresh dissolution media after every sample withdrawal. The collected samples were subjected to centrifugation at the rate of 13,800 rotations per minute for $30 \mathrm{~min}$ and the supernatant was lyophilized (Lab-conco Lyophilizer, USA) for a day. Dissolution was affected by dissolving the lyophilised, dried powder in methyl alcohol and this was analysed via RP-HPLC technique. The entire analysis was repeated thrice [18]. 


\subsection{Instigation of ischemia-reperfusion injury in spinal cord}

Induction of ischemia in spinal cord according previously published article. Mixture of ketamine (50 $\mathrm{mg} / \mathrm{kg}$ ) and xylazine (5 mg/kg) used as interperitoneal injection to anesthetize rats. Small aneurysm clip was placed between left kidney arteries and cross clamping used to cause ischemia. After surgical treatment animals were preyed on a common diet plan as well as water ad libitum. All animals were anesthetized and give up after $48 \mathrm{~h}$ of ischemia-reperfusion treatment. The 3, 4, 5 lumbar segments of spinal cord and blood samples were collected.

\subsection{Nitrite/Nitrate levels in blood plasma}

The UV-Vis spectrophotometer is used for determination of enzymatic conversion of nitrate to nitrite by nitrate reductase at $540 \mathrm{~nm}$. This shows nitrite/nitrate levels based on Griess reaction.

\subsection{Western blot analysis}

Total protein was extracted from tissues of treated and controlled group by utilizing a lysis buffer consisting of $1 \mathrm{M}$ Tris- $\mathrm{HCl}(\mathrm{pH} 7.5), 1 \mathrm{ml}$ 0.5M EDTA, NP-40 (1\% v/v), Trizma base with protease inhibitor. The protein concentration of the supernatant was identified making use of BCA as well as kept at $-80 \mathrm{C}$. The equivalent quantity ( $50 \mathrm{lg} /$ well) of proteins were divided in $10 \%$ SDS-PAGE, moved to a nitrocellulose membrane layer. The membrane layers were nurtured overnight at $4{ }^{\circ} \mathrm{C}$ with key antibodies of iNOS, $\mathrm{p}$ p38, overall p38 and $\beta$-actin. The equivalent amount of $\mathrm{IgG}$ antibodies in alkaline phosphatase were used for incubation for $2 \mathrm{~h}$ at RT. The membrane layers were established utilizing ECL reagent and protein signals evaluated by ChemiDoc after being normalizd to cognate $\beta$-actin signals.

\subsection{Quantification of advanced oxidation products (AOPP) and malondialdehyde (MDA)}

AOPP and MDA in lipid peroxidation were determined by following the reported method of Ohkawa. Briefly, Thiobarbituric acid (TBA) and trichloroacetic acid (TCA) was used for preparation of tissue homogenates and absorbance was recorded at $532 \mathrm{~nm}$. Addition of $\mathrm{MgCl}_{2}$ and phosphor tungstate to tissue homogenates helps in determining AOPP in the supernatant at $340 \mathrm{~nm}$ absorbance read in acidic conditions. The level of AOPP and MDA was shared as nM of MDA or AOPP reactants/100 $\mathrm{g}$ of wet tissues.

\subsection{Quantification of reduced glutathione (GSH)}


The elevated levels of glutathione were determined by previously reported method of Ellman. The tissue homogenates were combined with 5\% TCA and $0.3 \mathrm{M}$ disodium hydrogen phosphate. Add $1.0 \mathrm{ml}$ of Ellman's reagent to the homogenates mixture. Record the absorbance of sample against blank containing TCA at $420 \mathrm{~nm}$. The quantity of glutathione is expressed as $\mathrm{mg} / \mathrm{g}$ of protein.

\subsection{Quantification of superoxide dismutase (SOD) activity}

The SOD of samples was determined following by reported method by Misra and Fridovich. Briefly, 1.8 $\mathrm{mM}$ epinephrine was used for initiation of the response as well as absorbance read at $480 \mathrm{~nm}$. Non-polar solvents were added in tissue homogenates followed by $0.6 \mathrm{mM}$ EDTA solution as well as $0.1 \mathrm{M}$ carbonate buffer solution. The superoxide dismutase activity revealed as $50 \%$ inhibition of epinephrine in one minute/mg protein.

\subsection{Quantification of catalase (CAT) activity}

The CAT activity was performed by previously reported method by Takahara. Briefly, the tissue homogenate was mixed with $50 \mathrm{mM}$ phosphate buffer and absorbance was recorded by the addition of $30 \mathrm{mM} \mathrm{H}_{2} \mathrm{O}_{2}$ solution at $240 \mathrm{~nm}$. The enzyme activity was revealed as I moles of $\mathrm{H}_{2} \mathrm{O}_{2}$ decomposed $/ \mathrm{min} / \mathrm{mg}$ protein.

\subsection{Statistical analysis}

Analyses were performed in triplicate $(n=3)$ and the outcomes were expressed as mean \pm SD. For the statistical analysis the experimental data was tested by on-way analysis of variance (ANOVA). The values of $p<0.05$ were characteristic of statistical significance.

\section{Results}

\subsection{Characterization of RES-PUE loaded nanoparticles 3.1.1. Loading capacity and Drug entrapment efficiency}

The drug loading and entrapment efficiency of PLGA nanoparticles is depends on the solvent affinity of drug, polymer and surfactant. It's previously reported that PLGA have encapsulation efficiency of $82 \% \mathrm{w} / \mathrm{w}$. We have prepared five different RES-PUE loaded nanoparticles formulations taking different ratio of drug, PLGA and keeping same PVA content $[5,6]$. The RES-PUE loaded nanoparticles fabricated with a ratio of $1: 3$ drug-polymer shows, higher entrapment efficiency of $74.85 \%$ and loading capacity of 15.50\% (Table-1).

\subsubsection{Particle Size and Zeta potential of RES-PUE loaded nanoparticles}


The photon correlation spectroscopy is one of the easy and rapid method used for measurement of particle size. In RES-PUE loaded nanoparticles the mean particle size was ranges from $238 \mathrm{~nm}$ to $274 \mathrm{~nm}$ and particle size distribution was mono-dispersed (0.239 to 0.318 ) (Figure-1a). The stability of RES-PUE loaded nanoparticles in water can be attributed from single peak at particle size distribution plot $[5,6]$. Polymers and additives used in formulation influence the zeta potential of particle surface charge. Zeta potential of RES-PUE loaded nanoparticles was found to be $-12.6 \pm 2.1 \mathrm{mV}$ (Figure-1b).

\subsubsection{Scanning Electron Microscopy}

Scanning electron microscopic studies were performed for surface morphology and particle size measurement of nanoparticles. The SEM images of particles shown in Figure-1c, obtained directly by visualization through scanning electron microscope, so it appears smaller than the hydrodynamic layer obtained by Zetasizer.

\subsubsection{Atomic Force Microscopy}

Three dimensional surface morphology studies of the RES-PUE loaded nanoparticles were carried out by AFM on cleaned Si substrates (Figure-1d). The lateral sizes of the nanoparticles varies from image to image because of the variation in the tip shape [19]. The height of nanoparticle were varies from 9 to $11 \mathrm{~nm}$.

\subsubsection{Differential scanning calorimetry (DSC) studies}

The physical state of drug incorporated in PLGA nanoparticles was determined by DSC studies. The DSC curve of native Resveratrol, Puerarin, PLGA and RES-PUE loaded nanoparticles were shown in Figure-2a. The sharp endothermic peak at $262^{\circ} \mathrm{C}$ and $189^{\circ} \mathrm{C}$ in native Resveratrol and Puerarin respectively shows the melting point of native drug. The formulation mixture shows the endothermic peak at $183^{\circ} \mathrm{C}$, that of PLGA. In addition it shows less intense peak of Resveratrol and Puerarin, which may be due to surface bound drugs $[17,20]$.

\subsubsection{FTIR spectroscopy}

The FTIR spectrum of Resveratrol, Puerarin, PLGA and RES-PUE loaded nanoparticles were presented in Figure-2b. The FTIR spectrum of Resveratrol shows a major olefinic band at $965 \mathrm{~cm}-1$ and $3293 \mathrm{~cm}-1$ show due to $\mathrm{O}-\mathrm{H}$ stretching vibration. The band at $1383,1586 \mathrm{~cm}-1$ shows presence of $\mathrm{C}-0$ stretching in Resveratrol. Two characteristic peaks of puerarin at 1630 and $1260 \mathrm{~cm}-1$ shows presence of C-O stretching in puerarin.FTIR analysis of PLGA polymer showed distinct peaks at wave numbers $692 \mathrm{~cm}-1$, $1008 \mathrm{~cm}-1,1370 \mathrm{~cm}-1,1627 \mathrm{~cm}-1,1960 \mathrm{~cm}-1,2257 \mathrm{~cm}-1$. In addition peaks at $2441 \mathrm{~cm}-1$ and $3406 \mathrm{~cm}-1$ in both PLGA and RES-PUE loaded nanoparticles conforms the presence PLGA in the formulation. The presence of hydrogen bonding between hydroxyl groups of Resveratrol and carbonyl group of PLGA shifts $0-\mathrm{H}$ stretching band to higher wave number $[10,17,20]$.

\subsubsection{In vitro drug release studies}


In the present study cumulative percentage release data of drug from native Resveratrol, Puerarin and RES-PUE loaded nanoparticles divulges sustained release action of formulation (Figure-2c). The native Resveratrol and Puerarin shows $96 \%$ and $98 \%$ of drug release respectively in just 6 hrs of study, whereas the optimized formulation of RES-PUE loaded nanoparticles shows only $72 \%-79 \%$ of drug release over 36 hrs by diffusion mechanism.

\subsection{Pharmacokinetic study}

\subsubsection{Effect of RES-PUE loaded nanoparticles on plasma nitrite/nitrate levels}

RES-PUE loaded nanoparticles alter the circulating plasma nitrite/nitrate levels induced by reperfusion injury. The plasma nitrite/nitrate level in control group was less than the reperfusion injury induced group [21]. Administration of RES-PUE loaded nanoparticles decreases the nitrite/nitrate level in plasma of reperfusion injury induced rats (Figure-3a). However, the control group rats did not show any change in nitrite/nitrate levels in plasma even after treated with RES-PUE loaded nanoparticles.

\subsubsection{Effect of RES-PUE loaded nanoparticles on protein expressions}

Effect of RES-PUE loaded nanoparticles on IRI-induced, iNOS protein expression was measured by western blot. The protein (Figure-3b) expression was not altered in control, RES-PUE loaded nanoparticles and surgery groups [22]. The increased level of iNOS protein expression was normalizes after treatment with RES-PUE loaded nanoparticles in reperfusion injury induced rat groups.

\subsubsection{Effect of RES-PUE loaded nanoparticles on p38MAPK pathway}

Effect of RES-PUE loaded nanoparticles on reperfusion injury induced group was studied by activation of p38MAPK pathway indicating p38MAPK phosphorylation. As shown in Figure-4, the p38MAPK phosphorylation did not alter in control [16]. But, p38MAPK was distinctly phosphorylated in reperfusion injury induced group treated with RES-PUE loaded nanoparticles.

\subsubsection{Effect of RES-PUE loaded nanoparticles on Malondialdehyde and AOPP}

The level of Malondialdehyde (Figure-5a) and AOPP (Figure-5b) formed from lipid peroxidation/ oxidation was used to investigate the effect of RES-PUE loaded nanoparticles [23]. The reperfusion injury induced groups treated with RES-PUE loaded nanoparticles shows decreased level of Malondialdehyde and AOPP as compared to control group.

\subsubsection{Effect of RES-PUE loaded nanoparticles on GSH, SOD and CAT antioxidant activities}


The GSH non-enzymatic antioxidant activity level (Figure-6a) alters in RES-PUE loaded nanoparticles treated reperfusion injury induced group [13]. Similarly, the SOD (Figure-6b) and CAT (Figure-6c) enzymatic level didn't alter in control group, but significantly increases in RES-PUE loaded nanoparticles treated reperfusion injury induced group [11].

\section{Discussion}

The increase in Drug to polymer ratio increases the encapsulation efficiency. It was observed that increase in the drug to PLGA ratio from 20-40\% increases the drug encapsulation efficiency of nanoparticles. The increases in drug loading saturate the surface matrix if the PLGA nanoparticles, which favour the leaching, process of drug. So, the formulation with 1:3 drug to PLGA ratio was selected and used for all further studies.

The higher zeta potential value of the particles shows higher stability of nanoparticle formulations. Negative charge can be attributed tothe presence of free carboxyl groups of PLGA on the nanoparticle surface $[5,6]$. The projected PEG chains of PVA prevent aggregation of the particle despite of lower zeta potential. The SEM and AFM image of RES-PUE loaded nanoparticles was found to be spherical in shape with smoother surface $[6,14]$. The DSC and FTIR studies also reveal that Resveratrol and Puerarin were present as molecular dispersion form in the PLGA matrix.

In vitro drug release studies shows that the formulation shows a burst release of drug at initial stage ( $0.25 \mathrm{hrs}$ and $0.5 \mathrm{hrs}$ ), attributes the presence of surface bound drugs. Low melting point and hydrophobic nature of PLGA provides better sustained release action on solidification [17, 20].

In vivo pharmacokinetics studies shows no change in nitrite/nitrate levels and increased level of iNOS protein expression in plasma after treated with RES-PUE loaded nanoparticles. The reperfusion injury induced group treated with RES-PUE loaded nanoparticles shows decrease in p38 phosphorylation, Malondialdehyde and AOPP level as compared to control group.

Cytokines production and neutrophils activation causes loss of membrane lipid functions [24], which generates free radicals and results in cell death [25]. The free radical causes reoxygenation and changes the pathology of neurologic dysfunction in reperfusion injury induced group. The group treated with $10 \mathrm{mg} / \mathrm{kg}$ of RES-PUE loaded nanoparticles protects spinal cord from ischemia.

Oxidative stress induced in injured spinal cord tissues due to over production of nitric oxide. Similarly, several studies have been focused on inhibition of p38MAPK pathway by suppressing oxidative stress in reperfusion injury induced group $[3,26]$. Significant decrease in p38MAPK expression and plasma nitrite/nitrate levels were observed in RES-PUE loaded nanoparticles treated reperfusion injury induced group [27]. The Malondialdehyde and AOPP levels were in creased in reperfusion injury induced group and it correlated with cytokines levels [12]. 
The administration of RES-PUE loaded nanoparticles to reperfusion injury induced group significantly increases the antioxidant level i.e. GSH, SOD and CAT, which are acts as defence.

The multifunctional resveratrol and puerarin loaded nanoparticles were developed to enhance the oral bioavailability of resveratrol and puerarin. RES-PUE loaded nanoparticles protect spinal cord injured rat models. The RES-PUE loaded nanoparticle blocks the p38MAPK signalling pathway and expressed antioxidant effects. The further investigation may be acceptable to determine the efficacy of RES-PUE loaded nanoparticles on human spinal cord reperfusion injuries. Reperfusion injury induced free radicals were reduced by RES-PUE loaded nanoparticles treatment by increasing enzymatic and non-enzymatic antioxidant levels.

\section{Declarations}

\section{Ethics approval and consent to participate}

The animal experiment study protocol was approved by the institute's committee on animal research, Linyi central hospital, Shandong province, China. The legal and ethical approval was obtained prior to initiation of the research work on animals, and the experiments were performed in accordance with the animal ethical committee guidelines and regulations of Republic of China.

\section{Consent for publication}

Not applicable.

\section{Availability of data and material}

The supporting data for present findings are under ethics laws of university and is hence not presented here.

\section{Competing interests}

The authors declare no conflicts of interest.

\section{Funding}

This study was supported by funds from the Health Department of Shandong Province of China (grant nos. 2011QW008 and 2015WS0375).

\section{Authors' contributions}

WC, ZZ re associated planning the work The experiments were carried out by SZ, LZ whereas QS and LZ were also involved in data handling and processing. All the authors contributed equally in preparing the manuscript. All authors read and approved the final manuscript.

\section{Acknowledgements}

Page $11 / 20$ 
We express thanks to the staff and management of the Linyi central hospital, Shandong province, China.

\section{References}

1. Baumann MD, Kang CE, Tator CH, Shoichet MS (2010) Intrathecal delivery of a polymeric nanocomposite hydrogel after spinal cord injury. Biomaterials 31(30):7631-7639

2. Bremner P, Rivera D, Calzado MA, Obon C, Inocencio C, Beckwith C et al (2009) Assessing medicinal plants from South-Eastern Spain for potential anti-inflammatory effects targeting nuclear factorKappa B and other pro-inflammatory mediators. J Ethnopharmacol 124(2):295-305

3. Conti A, Miscusi M, Cardali S, Germano A, Suzuki H, Cuzzocrea S et al (2007) Nitric oxide in the injured spinal cord: synthases cross-talk, oxidative stress and inflammation. Brain Res Rev 54(1):205-218

4. Crowley-Weber CL, Dvorakova K, Crowley C, Bernstein H, Bernstein C, Garewal H et al (2003) Nicotine increases oxidative stress, activates NF-kappaB and GRP78, induces apoptosis and sensitizes cells to genotoxic/xenobiotic stresses by a multiple stress inducer, deoxycholate: relevance to colon carcinogenesis. Chem Biol Interact 145(1):53-66

5. Chou TC (2010) Drug combination studies and their synergy quantification using the Chou-Talalay method. Cancer Res 70(2):440-446

6. Mohanty C, Acharya S, Mohanty AK, Dilnawaz F, Sahoo SK (2010) Curcumin-encapsulated MePEG/PCL diblock copolymeric micelles: a novel controlled delivery vehicle for cancer therapy. Nanomedicine (Lond) 5(3):433-449

7. Bhaumik S, Anjum R, Rangaraj N, Pardhasaradhi BV, Khar A (1999) Curcumin mediated apoptosis in AK-5 tumor cells involves the production of reactive oxygen intermediates. FEBS Lett 456(2):311314

8. Terada H, Kazui T, Takinami M, Yamashita K, Washiyama N, Muhammad BA (2001) Reduction of ischemic spinal cord injury by dextrorphan: comparison of several methods of administration. $J$ Thorac Cardiovasc Surg 122(5):979-985

9. Frémont L (2000) Biological effects of resveratrol. Life Sci 66:663-673

10. Song Y, Liu J, Zhang F, Zhang J, Shi T, Zeng Z (2013) Antioxidant effect of quercetin against acute spinal cord injury in rats and its correlation with the p38MAPK/iNOS signaling pathway. Life Sci 92(24-26):1215-1221

11. Misra HP, Fridovich I (1972) The role of superoxide anion in the autoxidation of epinephrine and a simple assay for superoxide dismutase. J Biol Chem 247(10):3170-3175

12. Pekarkova I, Parara S, Holecek V, Stopka P, Trefil L, Racek J et al (2001) Does exogenous melatonin influence the free radicals metabolism and pain sensation in rat? Physiol Res 50(6):595-602

13. Takahara S, Hamilton HB, Neel JV, Kobara TY, Ogura Y, Nishimura ET (1960) Hypocatalasemia: a new genetic carrier state. J Clin Invest 39:610-619 
14. Mohanty AK, Mohanta GP: Dual anticancer drug loaded methoxy poly (ethylene glycol)-poly ( $\varepsilon$ caprolactone) block copolymeric micelles as novel drug carriers. Int J Pharm Pharm Sci 2014:In press

15. Cruise GM, Scharp DS, Hubbell JA (1998) Characterization of permeability and network structure of interfacially photopolymerized poly(ethylene glycol) diacrylate hydrogels. Biomaterials 19(14):12871294

16. Ohkawa H, Ohishi N, Yagi K (1979) Assay for lipid peroxides in animal tissues by thiobarbituric acid reaction. Anal Biochem 95(2):351-358

17. Mohanty AK, Dilnawaz F, Mohanty C, Sahoo SK (2010) Etoposide-loaded biodegradable amphiphilic methoxy (poly ethylene glycol) and poly (epsilon caprolactone) copolymeric micelles as drug delivery vehicle for cancer therapy. Drug Deliv 17(5):330-342

18. Muthukkumar S, Ramesh T, Bondada S (1995) Rapamycin, a potent immunosuppressive drug, causes programmed cell death in B lymphoma cells. Transplantation 60(3):264-270

19. Dunne M, Corrigan I, Ramtoola Z (2000) Influence of particle size and dissolution conditions on the degradation properties of polylactide-co-glycolide particles. Biomaterials 21(16):1659-1668

20. Sahoo SK, Ma W, Labhasetwar V (2004) Efficacy of transferrin-conjugated paclitaxel-loaded nanoparticles in a murine model of prostate cancer. Int J Cancer 112(2):335-340

21. Lafci G, Gedik HS, Korkmaz K, Erdem H, Cicek OF, Nacar OA et al (2013) Efficacy of iloprost and montelukast combination on spinal cord ischemia/reperfusion injury in a rat model. $J$ Cardiothorac Surg 8:64

22. Lemmon VP, Ferguson AR, Popovich PG, Xu XM, Snow DM, Igarashi M et al (2014) Minimum information about a spinal cord injury experiment: a proposed reporting standard for spinal cord injury experiments. J Neurotrauma 31(15):1354-1361

23. Ellman GL (1959) Tissue sulfhydryl groups. Arch Biochem Biophys 82(1):70-77

24. Sa G, Das T (2008) Anti cancer effects of curcumin: cycle of life and death. Cell Div 3:14

25. Mackay ME, Tuteja A, Duxbury PM, Hawker CJ, Van Horn B, Guan Z et al (2006) General strategies for nanoparticle dispersion. Science 311 (5768):1740-1743

26. Maggio DM, Chatzipanteli K, Masters N, Patel SP, Dietrich WD, Pearse DD (2012) Acute molecular perturbation of inducible nitric oxide synthase with an antisense approach enhances neuronal preservation and functional recovery after contusive spinal cord injury. J Neurotrauma 29(12):22442249

27. Sgaravatti AM, Magnusson AS, Oliveira AS, Mescka CP, Zanin F, Sgarbi MB et al (2008) Effects of 1,4butanediol administration on oxidative stress in rat brain: study of the neurotoxIRIty of gammahydroxybutyric acid in vivo. Metab Brain Dis 24(2):271-282

\section{Table}


Table-1: Physico-chemical characterization of RES-PUE loaded nanoparticles (data represents mean \pm SD).

\begin{tabular}{|llll|}
\hline Batch No. & Drug-Polymer ratio & $\begin{array}{l}\text { Loading capacity } \\
(\% \mathbf{w} / \mathbf{w})^{\mathbf{a}}\end{array}$ & Entrapment efficiency $(\% \mathbf{w} / \mathbf{w})^{\mathbf{b}}$ \\
\hline RES-PUE-NP1 & $1: 2$ & $21.91 \pm 0.09$ & $69.63 \pm 0.68$ \\
\hline RES-PUE-NP2 & $1: 3$ & $15.50 \pm 0.08$ & $74.85 \pm 0.89$ \\
\hline RES-PUE-NP3 & $1: 4$ & $10.71 \pm 0.86$ & $72.72 \pm 0.80$ \\
\hline RES-PUE-NP4 & $1: 5$ & $8.60 \pm 0.17$ & $61.43 \pm 0.64$ \\
\hline RES-PUE-NP5 & $1: 6$ & $7.78 \pm 0.20$ & $54.24 \pm 0.42$ \\
\hline
\end{tabular}

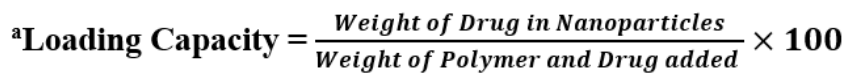

${ }^{\mathrm{b}}$ Entrapment efficiency $=\frac{\text { Weight of Drug in Nanoparticles }}{\text { Weight of Drug added }} \times 100$

\section{Figures}



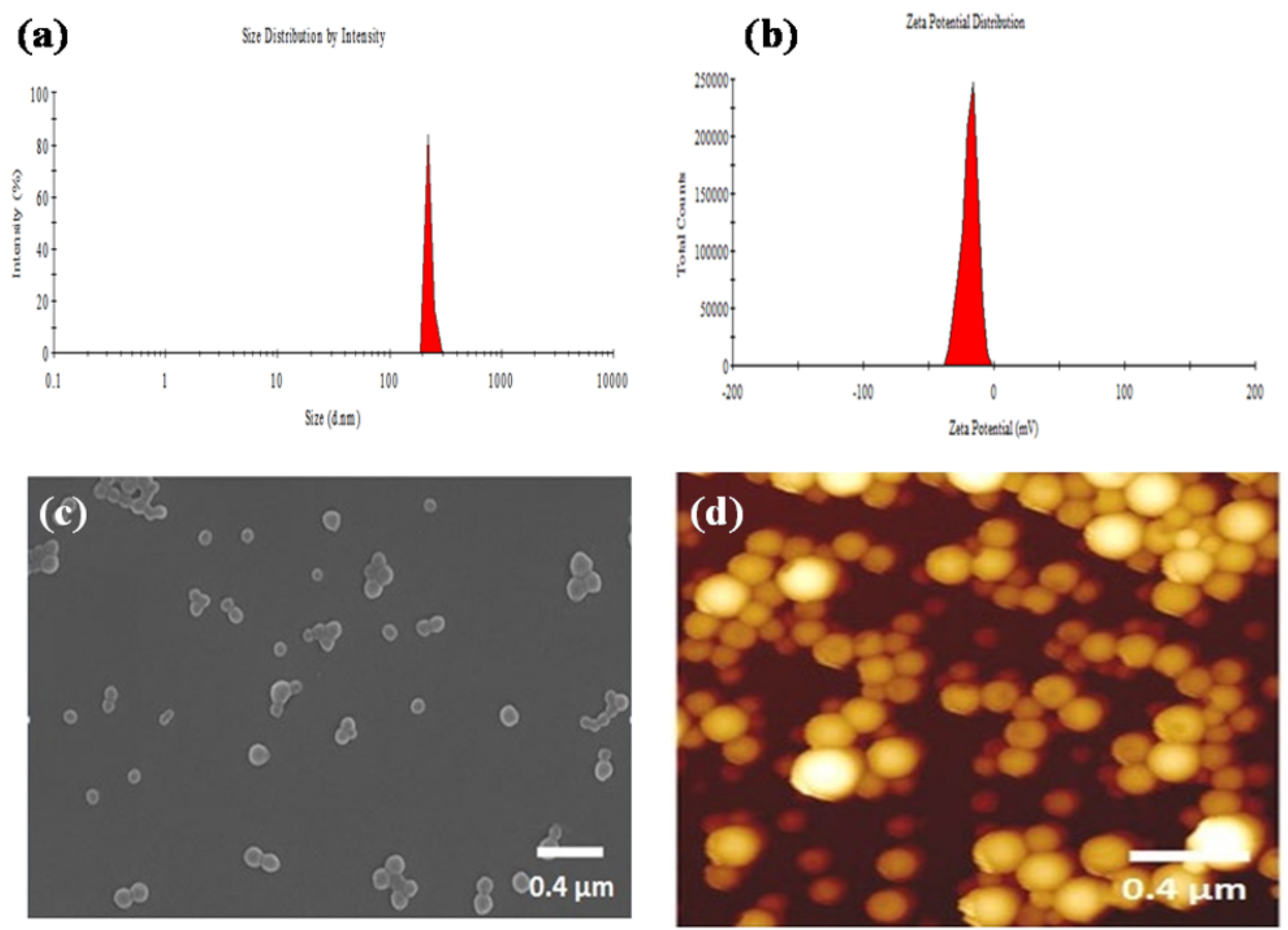

\section{Figure 1}

Surface morphology of RES-PUE loaded nanoparticles(a) Mean particle size distributions of RES-PUE loaded nanoparticles, (b) Zeta potential of RES-PUE loaded nanoparticles, showing negative values, (c) Scanning electron microscopyimages of RES-PUE loaded nanoparticles (bar $=400 \mathrm{~nm}$ ). (d) Atomic force microscopyimages of RES-PUE loaded nanoparticles $($ bar $=400 \mathrm{~nm})$. 
(a)

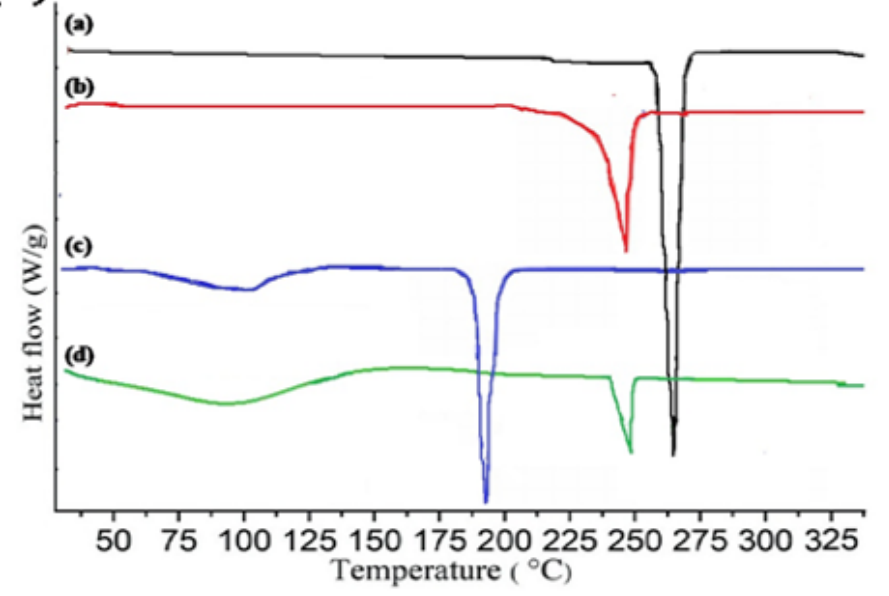

(b)
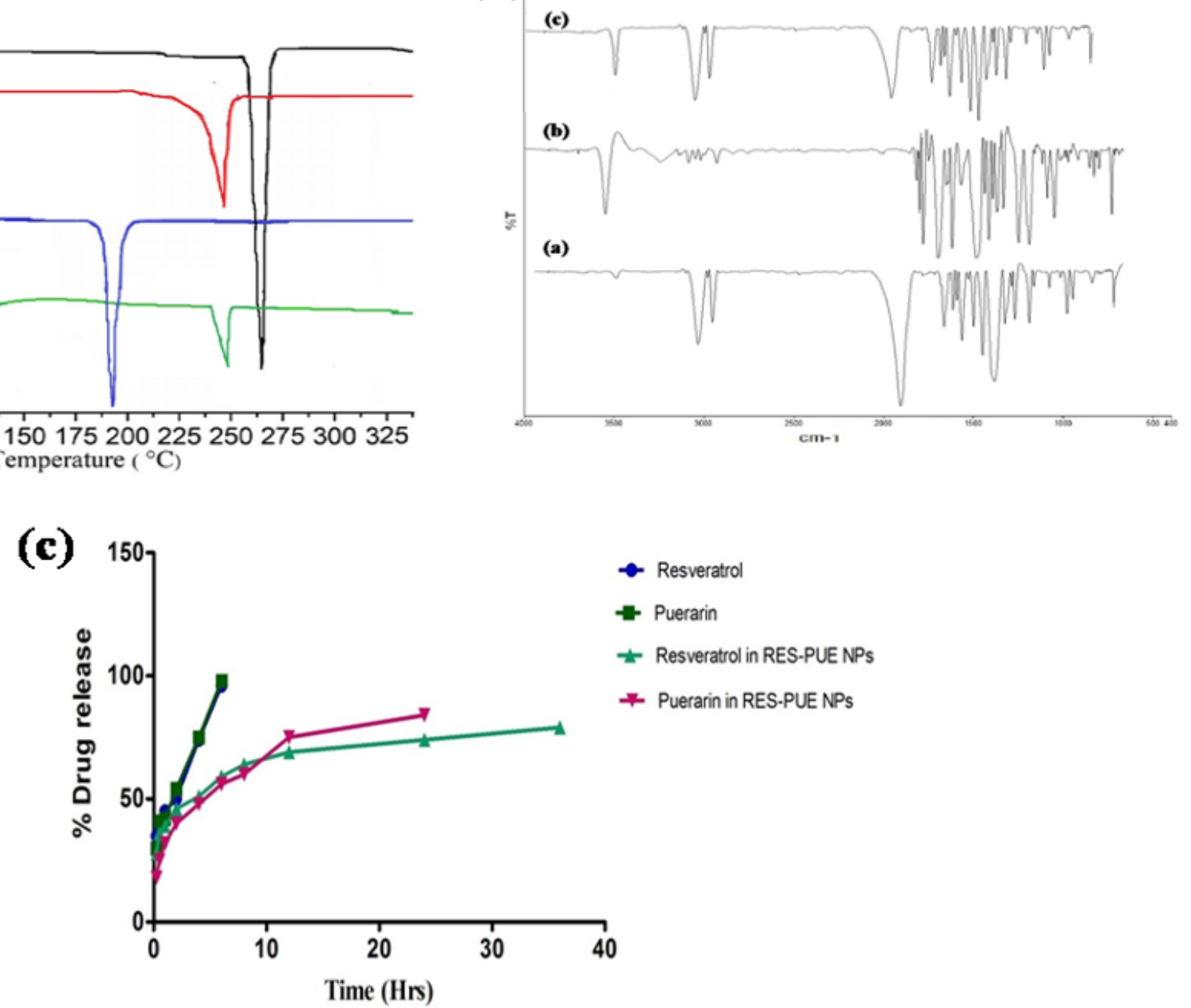

\section{Figure 2}

(a): Differential scanning calorimetry (DSC) thermogram of (a) Native resveratrol, (b) PLGA, (c) Native puerarin and (d) RES-PUE loaded nanoparticles. (b): Fourier transforms infrared spectra of (a) Native resveratrol, (b) Native puerarin and (c) RES-PUE loaded nanoparticles. (c): In vitro drug release study of Native resveratrol, Native puerarin and RES-PUE loaded nanoparticles. Data as mean \pm standard error ofmean $(n=3)$. 
(a)

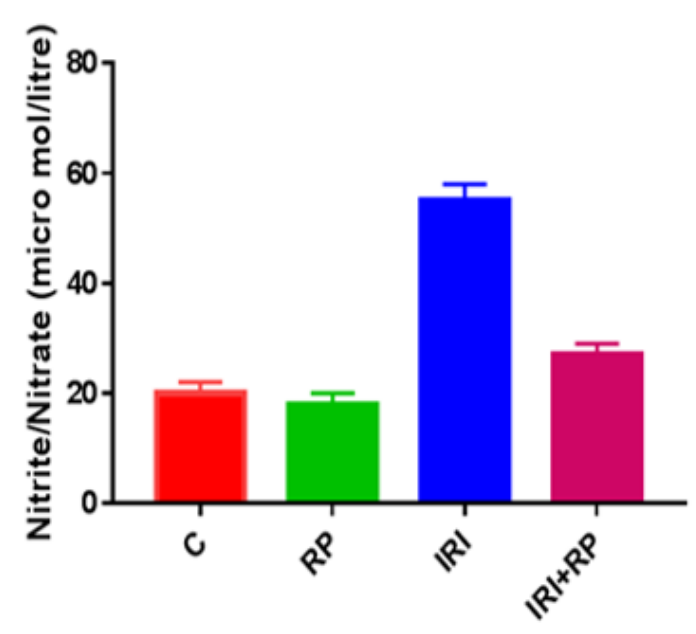

(b)

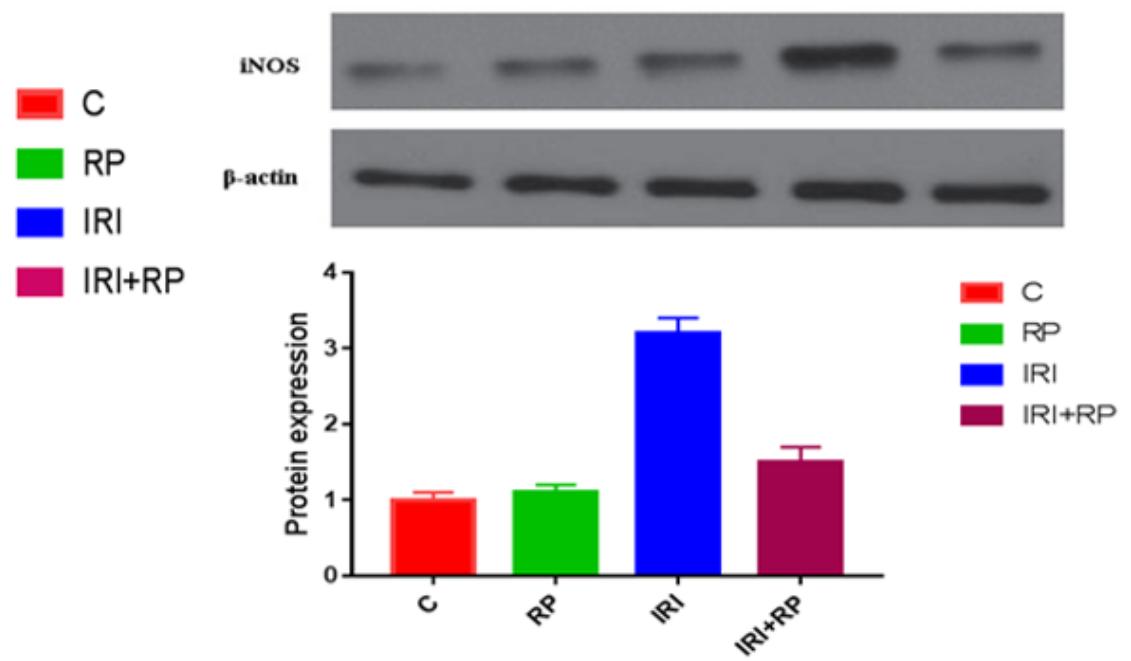

Figure 3

Effect of RES-PUE loaded nanoparticles on plasma nitrite/nitrate and protein expressions. (a) Estimation of levels of nitrite/nitrate in rats plasma. (b) $\beta$-actin normalized protein levels in spinal cord tissue homogenates. (C: Control, RP: Resveratrol and Puerarin nanoparticles, IRI: Ischemia-reperfusion injury, IRI + RP: Ischemia-reperfusion injury treated with Resveratrol and Puerarin nanoparticles) $\mathrm{P}$ value $<0.001$ compared with IRI group 
c

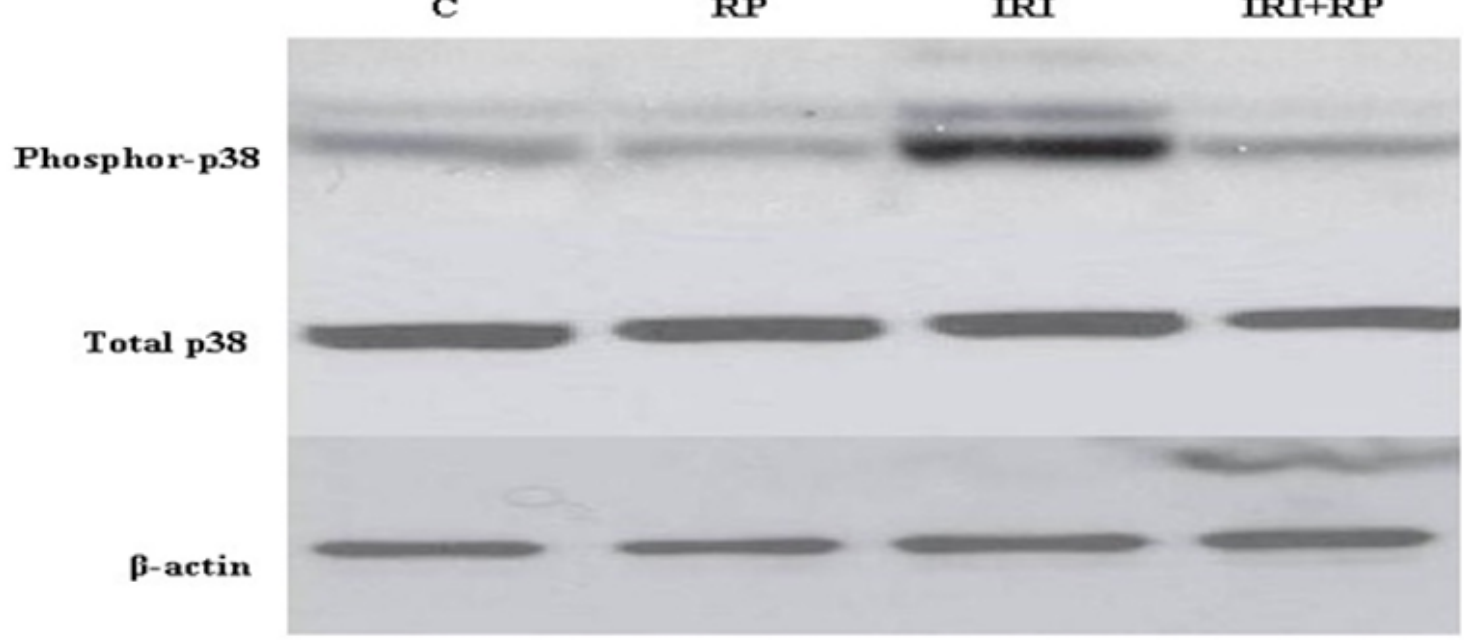

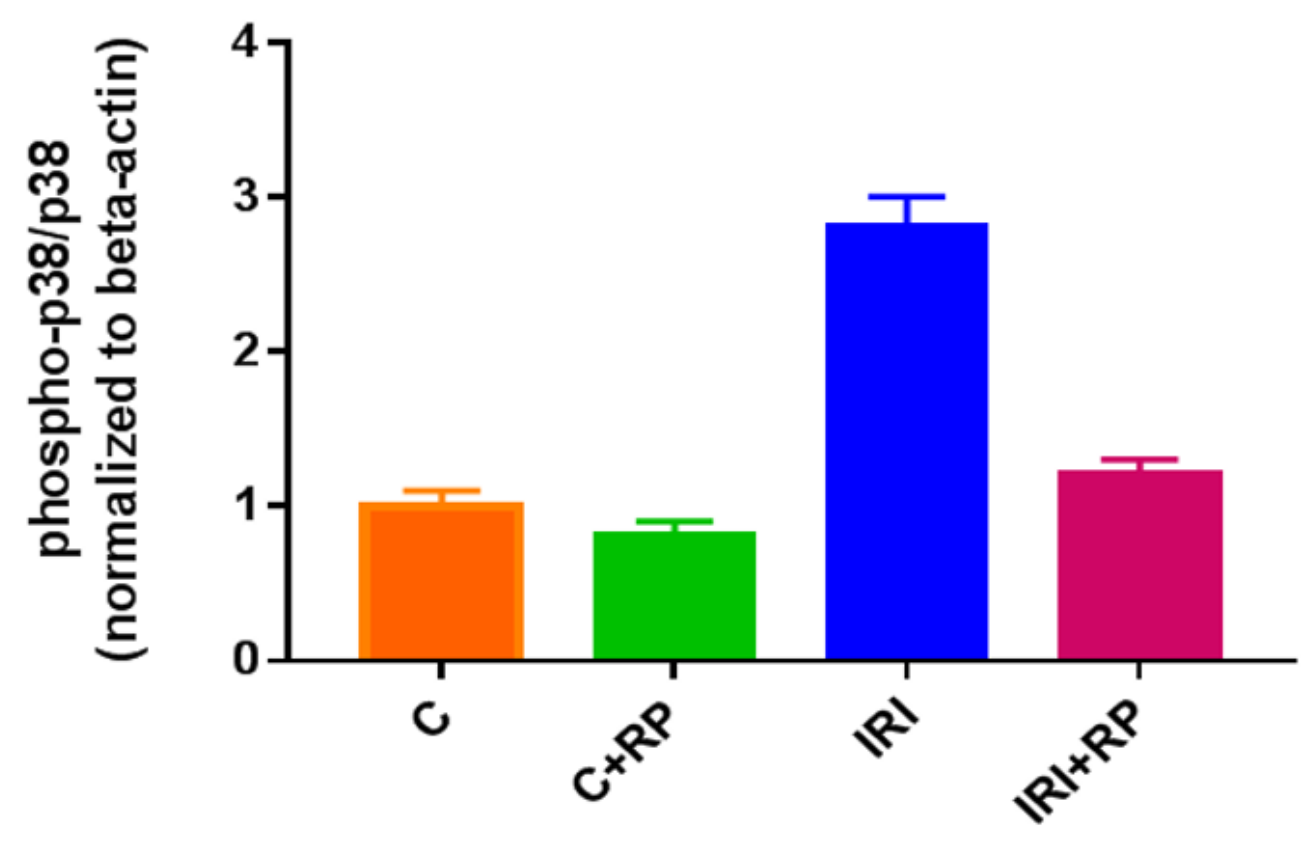

Figure 4

Effect of RES-PUE loaded nanoparticles on p38MPAK pathway. Estimation of protein expressions of phospho-p38 MPAK and total MAPK in spinal cord tissue homogenates. (C: Control, RP: Resveratrol and Puerarin nanoparticles, IRI: Ischemia-reperfusion injury, IRI + RP: Ischemia-reperfusion injury treated with Resveratrol and Puerarin nanoparticles) P value $<0.001$ compared with IRI group 
(a)

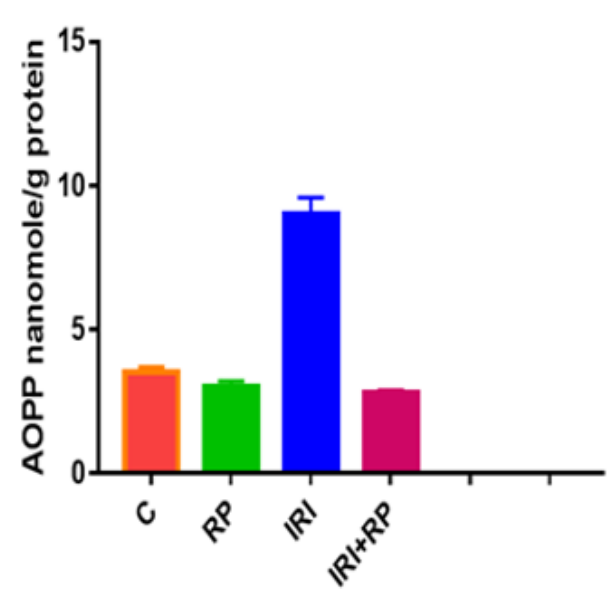

(b)

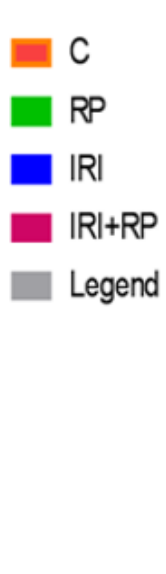

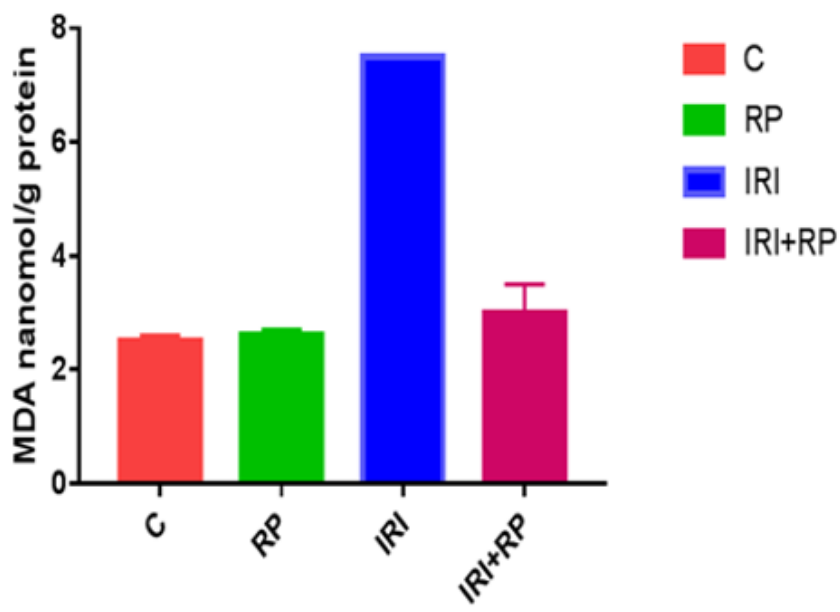

Figure 5

Effect of RES-PUE loaded nanoparticles on lipid peroxidation (MDA) and oxidation (AOPP) production. (a) Malondialdehyde (MDA) and (b) Advanced oxidation products (AOPP) in spinal cord tissue homogenates. (C: Control, RP: Resveratrol and Puerarin nanoparticles, IRI: Ischemia-reperfusion injury, IRI + RP: Ischemia-reperfusion injury treated with Resveratrol and Puerarin nanoparticles) $P$ value $<0.001$ compared with IRI group 
(a)

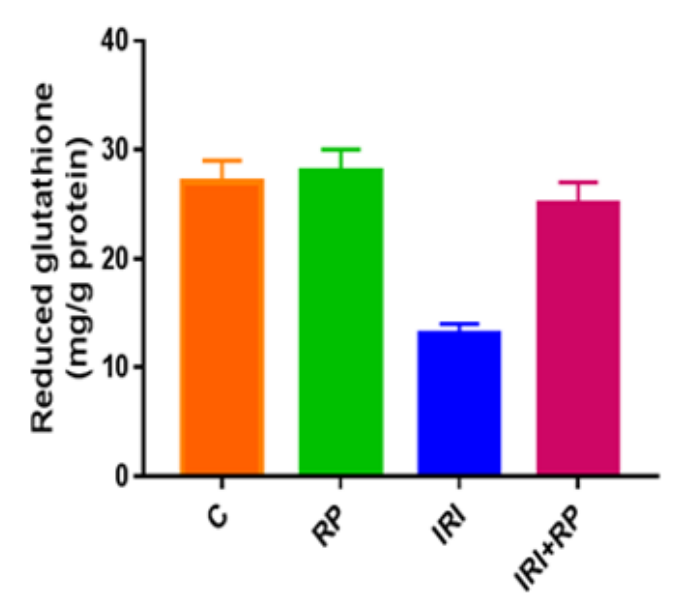

(b)

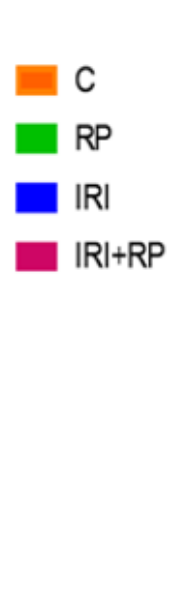

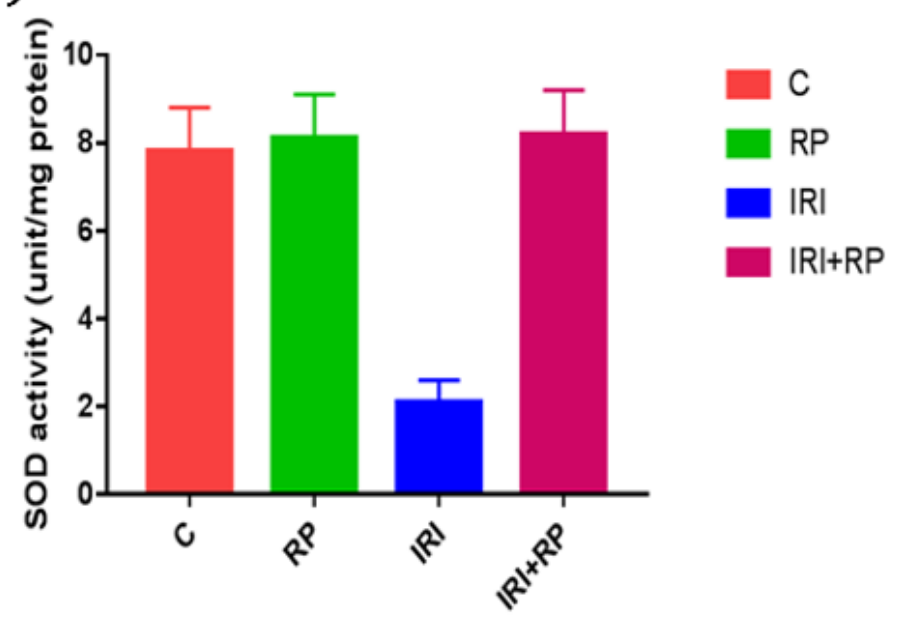

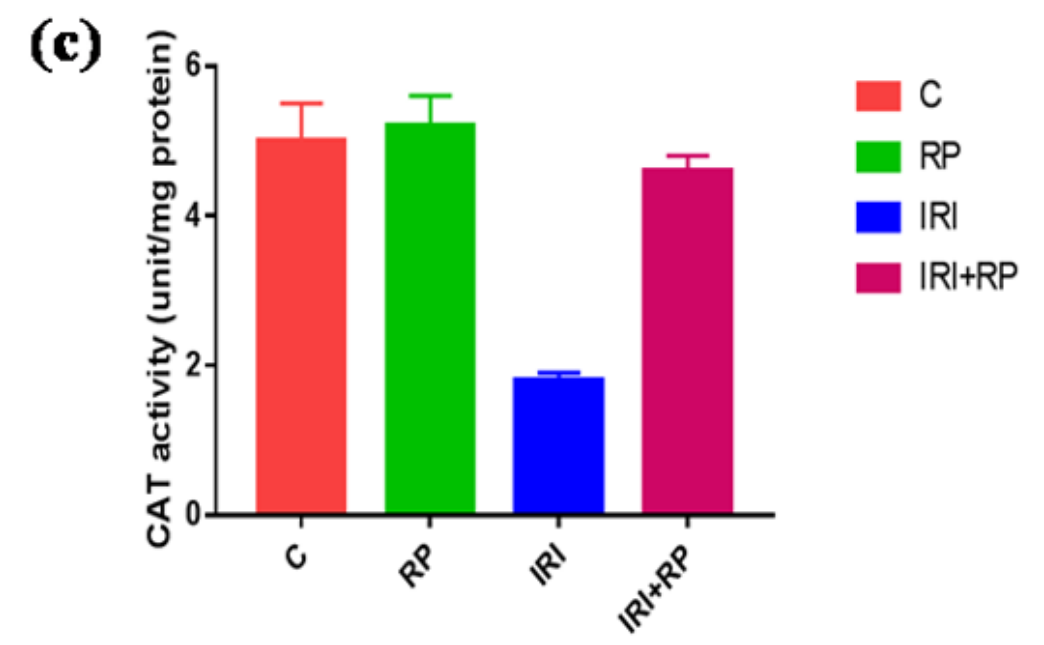

Figure 6

Effect of RES-PUE loaded nanoparticles on the level of reduced GSH, SOD and CAT. (a) Level of reduced glutathione (GSH), (b) Activity of SOD enzyme and (c) Activity of CAT enzyme levels in spinal cord tissue homogenates.(C: Control, RP: Resveratrol and Puerarin nanoparticles, IRI: Ischemia-reperfusion injury, IRI + RP: Ischemia-reperfusion injury treated with Resveratrol and Puerarin nanoparticles) $P$ value $<0.001$ compared with IRI group. 